Revue internationale P.M.E.

Économie et gestion de la petite et moyenne entreprise

\title{
La construction d'une capacité de réponse d'une PMI à l'international : une mise à l'épreuve des faits du modèle de Bilkey et Tesar
}

\section{Blandine Ageron}

Volume 14, numéro 2, 2001

URI : https://id.erudit.org/iderudit/1008690ar

DOI : https://doi.org/10.7202/1008690ar

Aller au sommaire du numéro

Éditeur(s)

Presses de l’Université du Québec

ISSN

0776-5436 (imprimé)

1918-9699 (numérique)

Découvrir la revue

Citer cet article

Ageron, B. (2001). La construction d'une capacité de réponse d'une PMI à l'international : une mise à l'épreuve des faits du modèle de Bilkey et Tesar. Revue internationale P.M.E., 14(2), 9-39. https://doi.org/10.7202/1008690ar
Résumé de l'article

L'objectif de cet article est de décrire et de comprendre le processus d'exportation de la PMI et la structuration du processus d'internationalisation d'une PMI. Ce travail s'appuie sur une étude empirique réalisée auprès d'une PMI exportatrice. 


\title{
La construction d'une capacité de réponse d'une PMI à l'international : une mise à l'épreuve des faits du modèle de Bilkey et Tesar
}

\author{
Blandine AGERON \\ Université Jean-Moulin Lyon III
}

\begin{tabular}{c} 
MOTS CLÉS \\
\hline PMI - Processus d'internationalisation - Modèles behavioristes \\
Dirigeant - Cohérence - Logiques industrielles et / ou sociales \\
Outil d'aide à la conception
\end{tabular}

\section{RÉSUMÉ}

L'objectif de cet article est de décrire et de comprendre le processus d'exportation de la PMI et la structuration du processus d'internationalisation d'une PMI. Ce travail s'appuie sur une étude empirique réalisée auprès d'une PMI exportatrice.

\section{L'AUTEURE}

BLANDINE AGERon est enseignante-chercheure au sein de l'équipe EURISTIK (Unité mixte de recherche no 5055 du CNRS) de l'Université Jean-Moulin Lyon III. Ses thèmes de recherche sont 1) le questionnement et la mise en cohérence des logiques industrielles et / ou sociales de la PMI dans un contexte d'internationalisation, 2) la compréhension de la dynamique de structuration du processus d'internationalisation de la petite entreprise et 3) le questionnement des modèles behavioristes existants et les enrichissements théoriques et empiriques autour du thème du processus d'internationalisation. En 1999, elle a terminé sa thèse de doctorat à I'Université Jean-Moulin Lyon III Processus d'internationalisation de la PMI : un outil d'aide à la conception (et au pilotage) sous la direction du professeur Alain-Charles Martinet. Adresse : EURISTIK, Unité mixte de recherche n 5055 du CNRS, Université Jean-Moulin Lyon III, 15, quai Claude-Bernard, B.P. 0638, 69239 Lyon, cedex 02, France. Courriel : <Blandine.Ageron @univ-lyon3.fr>. 


\begin{abstract}
The aim of this article is to describe and to understand the export process of small businesses and the structuration process of international activity of a small company. This work relies on an empirical investigation, case study, in a small business.

\section{RESUMEN}

El objetivo de este articulo es describir y entender del proceso de exportación de las pequeñas y medianas empresas, la estructuración del proceso de internacionalización de las PyMEs. Este trabajo se funda en un estudio empirico realizado con las PyMEs exportadoras.
\end{abstract}

\title{
ZUSAMMENFASSUNG
}

Ziel dieses Artikel ist es, die Exportprozesse von kleinen und mittleren Industrieunternehmen sowie die Struktur ihrer Internationalisierungsprozesse zu beschreiben und zu verstehen. Diese Arbeit Stützt sich auf eine empirische Studie, die bei exportorientierten kleinen und mittleren Industrieunternehmen realisiert wurde.

\section{Introduction}

La plupart des études empiriques portant sur les processus d'internationalisation des PME cherchent à identifier les caractéristiques spécifiques qui permettent de différencier les activités à l'export des entreprises. Rares sont celles qui essayent de décrire et de comprendre la dynamique de structuration du processus d'internationalisation. Or, un tel travail nous semble nécessaire, et ce, pour plusieurs raisons :

- pour comprendre le processus d'enchaînement des stades d'exportation pour l'ensemble des modèles issus de la théorie du cycle de vie (Johanson et Wiedersheim-Paul, 1975 ; Johanson et Vahlne, 1977);

- pour saisir une modélisation des variables structurantes du processus d'internationalisation et proposer une base de connaissances de ce processus ;

- pour aider le dirigeant à raisonner et à concevoir son propre processus d'internationalisation, indépendamment d'un cheminement prédéfini (passage à travers différents stades), comme le laissent entendre la plupart des modèles behavioraux.

Dans cet article, nous nous attardons non seulement à l'origine de l'exportation d'une PMI, mais également aux modifications structurelles, organisationnelles et industrielles conséquentes.

Notre recherche repose sur l'étude monographique d'une PMI, dont l'activité à l'export s'est intensifiée au fil des ans. Ayant construit son activité à l'international, 
au cours de son développement, l'entreprise affiche aujourd'hui un objectif en matière d'export : «développer d'ici à deux ans l'activité internationale [...] afin que celle-ci atteigne $50 \%$ de l'activité du territoire national ». Cette exportation n'a pourtant pas été immédiate : elle est le résultat d'un long processus séquentiel construit au fur et à mesure du développement de l'entreprise (la PMI, occupée tout d'abord localement, s'est par la suite intéressée à son marché régional puis national et, enfin, international). En d'autres termes, l'activité d'exportation est le résultat, de la part de la PMI, d'une construction d'une capacité de réponse ${ }^{1}$ à ses marchés à l'international.

Si l'hypothèse selon laquelle l'exportation est un phénomène séquentiel n'est pas nouvelle (Reid, 1981), l'idée de construction d'une capacité de réponse de la PMI à des marchés à l'international rompt avec les nombreux travaux qui ont été menés jusqu'à présent dans ce champ. Le processus d'exportation ou la performance à l'export ne s'explique plus par une relation causale entre ce phénomène et un ensemble de variables, managériales, organisationnelles et décisionnelles, mais dans la structuration de l'entreprise elle-même.

L'objectif du travail présenté est de rendre compte de la construction de cette capacité de réponse à partir d'un modèle synthétique de la décision d'exporter; modèle que nous mettons ensuite à l'épreuve des faits à travers l'étude monographique d'une PMI. Subséquemment, nous montrons de façon beaucoup plus générale que les modèles issus de la théorie des stades (modèles behavoriaux) constituent un point d' ancrage pertinent et utile pour saisir, expliquer et comprendre le processus d'internationalisation de la petite entreprise.

\section{La PMI et l'international}

\subsection{La PMI et l'exportation}

La littérature sur le comportement exportateur de la PME renvoie à deux courants de recherche parallèles.

\subsubsection{Variables explicatives de l'internationalisation des entreprises}

Le premier courant s' attache à étudier les relations causales existant entre le comportement exportateur d'une PMI et un ensemble de variables externes et internes.

Deux optiques de recherche s'inscrivent dans ce courant:

- la première s'attache à démontrer la prédominance d'une variable sur le comportement exportateur de la PMI (Roux, 1987);

1. Le terme de «capacité de réponse » est défini plus loin dans le texte.

Revue internationale P.M.E., vol. 14, nº 2, 2001

(C) 2001 - Presses de l'Université du Québec

Édifice Le Delta I, 2875, boul. Laurier, bureau 450, Sainte-Foy, Québec G1V 2M2 - Tél. : (418) 657-4399 - www.puq.uquebec.ca

Tiré de : Revue internationale P.M.E., vol. $14, \mathrm{n}^{\circ} 2$, sous la direction de Pierre-André Julien. 
- la seconde cherche à cerner les différences entre des PMI exportatrices et des PMI non exportatrices (Yaprak, 1985 ; Keng et Jiuan, 1989).

Le travail de synthèse de Miesenbock (1988) propose une revue importante de la littérature sur le thème «PMI et exportation». Le travail de présentation et de comparaison des études empiriques qu'il a pu trouver sur ce thème l'a amené à distinguer deux types de variables influençant la décision d'exporter : des variables clés et des variables accessoires.

Les variables clés. Traditionnellement caractérisées par le décideur (Roux, 1987), le produit (Kleinschmidt et Cooper, 1984), le pays d'origine (Tesar et Tarleton, 1982) et les stimuli à l'export (O'Rourke, 1989), ces variables engendrent une forte relation causale avec le comportement exportateur ou non de l'entreprise.

Les variables accessoires. Elles correspondent souvent aux barrières à l'exportation (Cavusgil, 1982; Bilkey et Tesar, 1977), aux caractéristiques démographiques de la PMI (Yaprak, 1985), aux caractéristiques managériales (Kleinschmidt et Cooper, 1984) et aux variables marketing (Cavusgil, 1982 ; Bilkey et Tesar, 1977). Ces variables n'ont qu'une relation positive ou négative, sans causalité explicite.

\section{A. Les variables clés}

1. Le décideur ou les caractéristiques individuelles du dirigeant. Toutes les études empiriques sur le comportement à l'export du dirigeant distinguent les caractéristiques psychologiques du décideur (attitudes envers l'export) des caractéristiques objectives (voyages à l'étranger, langues parlées). Dichtl et al. (1984) écrivent, à ce propos, que l'exportation débute, habituellement, dans un pays proche culturellement ; l'exportateur parle plus de langues que le non-exportateur et il est plus dynamique et réactif.

2. Le produit est certainement, avec le comportement du décideur, la variable clé qui pèse le plus dans la décision d'exporter. Traduction matérielle du comportement exportateur de la PMI, cet élément conditionne le succès ou l'échec de l'exportation. Dans un article sur les stratégies compétitives des PMI italiennes, Lanzara (1987) examine les facteurs qui ont permis à ces entreprises d'atteindre et de conserver une compétitivité internationale. L'auteur montre comment ces entreprises ont su développer des stratégies (de prix faibles, de différenciation du produit, d'adaptation du produit et d'innovation technologique) adaptées à l'environnement pour accroître leur présence internationale.

3. Le pays d'origine de la PMI exportatrice. Certains systèmes légaux facilitent (avantages de taxe à l'export) ou compliquent (réglementation à l'export) les affaires internationales. De même, les infrastructures (facilités de distribution), le système éducatif et les mentalités ont une influence sur 
le comportement exportateur de la PMI. Tesar et Tarleton (1982) soulignent, à cet égard, dans une étude réalisée auprès de PMI des États du Wisconsin et de la Virginie, les différences de comportements relevées entre les entreprises de ces deux États, du fait de ces différences de pratiques entre États.

4. Les stimuli à l'export sont regroupés en deux catégories :

- les stimuli internes : les excédents de capacité, les avantages technologiques et marketing, etc. ;

- les stimuli externes : les commandes spontanées, la saturation du marché domestique, la pression de la concurrence, etc.

\section{B. Les variables accessoires}

1. Les barrières à l'export. Généralement, on distingue deux types de barrières :

- les barrières internes : la capacité industrielle insuffisante, l'information insuffisante, les difficultés liées à la distribution, etc. ;

- les barrières externes : l'absence de demande perçue de l'étranger, les coûts à l'exportation, etc.

\section{Les caractéristiques démographiques de la PMI :}

- la taille de la PMI qui, si elle est trop petite, est souvent perçue comme un handicap pour l'exportation (Bonaccorsi, 1992 ; Reid, 1982). À cet effet, on peut noter que la dépendance de la PMI à l'égard d'autres entreprises de plus grande taille, voire de groupes, constitue également un élément « facilitateur » de l'engagement à l'international de la PMI ;

- l'âge de l'entreprise. Les jeunes entreprises sont fréquemment plus actives à l'exportation que leurs homologues créées depuis plusieurs années et qui restreignent en général leur territoire d'échange au niveau national.

3. Les caractéristiques managériales de l'entreprise : certains auteurs (Robinson et al., 1986; Fankam, 1998) ont trouvé une relation positive entre la planification à long terme et le comportement exportateur de la PMI. D’autres (Langston et Teas, 1976 ; Kalika, 1986) ont montré qu'un personnel qualifié et le nombre de langues parlées par les responsables est également un gage de réussite à l'international.

4. Les variables marketing comme la qualité, le design, la promotion, la publicité, la participation aux foires et, plus généralement, les variables du marketing mix participent du succès ou de l'échec de certaines PMI à l'international.

Revue internationale P.M.E., vol. 14, n 2, 2001 


\subsubsection{Stades d'internationalisation des entreprises}

Le second courant est caractérisé par les modèles ${ }^{2}$, issus de la théorie du cycle de vie, développés sur le processus d'exportation de l'entreprise. Les auteurs considèrent l'évolution de l'internationalisation comme un processus d'apprentissage comportant des étapes que franchit l'entreprise.

Le premier modèle, appelé modèle Uppsala (Johanson et Vahlne, 1977), décline le développement à l'export de l'entreprise selon des modes d'engagement successifs et incrémentaux à l'étranger.

Le second modèle, appelé modèle Innovation, considère l'exportation comme une innovation au sein de la PMI.

\section{A. Le modèle Uppsala}

Développé par l'école nordique d'Helsinki, ce modèle distingue quatre stades successifs d'engagement sur des marchés étrangers ${ }^{3}$.

Stade 1: absence d'activité à l'export régulière.

Stade 2: une activité d'exportation étayée par la mise en place d'un réseau de représentants indépendants (agents).

Stade 3: l'établissement d'une filiale de vente à l'étranger.

Stade 4: la mise en place d'unités de fabrication à l'étranger.

Pour expliquer l'exportation vers de nouveaux marchés, les auteurs font l'hypothèse que les entreprises pénètrent de nouveaux marchés avec, successivement, une distance psychique ${ }^{4}$ plus importante. L'entreprise intégrant et apprenant de ses expériences passées, elle est capable d'utiliser ces apprentissages pour accroître son engagement sur des marchés étrangers, ce qui entraîne une réduction de la distance psychique qui la sépare de nouveaux territoires étrangers.

Le caractère incrémental est expliqué par un modèle dynamique développé par les auteurs. La structure principale de ce modèle fait la distinction entre, d'une part, un aspect statique, représenté par l'engagement sur le marché (ressources engagées) et la connaissance des marchés étrangers et, d'autre part, un aspect dynamique, renvoyant aux décisions d'engager les ressources et à la performance des activités présentes.

2. Pour une revue relativement exhaustive des modèles voir Leonidou et Katsikeas (1996).

3. Chacun des stades successifs représente un degré supérieur d'engagement à l'international.

4. Cette notion de distance psychique renvoie à des facteurs modifiant ou empêchant l'échange d'information entre le marché et l'entreprise (différence de langage, de système éducatif, etc.). 


\section{B. Le modèle Innovation}

La perspective d'innovation pour expliquer l'exportation a été retenue dans différents modèles, les plus connus étant ceux de Bilkey et Tesar (1977), Cavusgil (1980), Czinkota (1982) et Reid (1981) et plus récemment Moon et Lee (1990) et Crick (1995). Tous ces modèles traitent du comportement exportateur des PMI ; les seules différences se situent au niveau du nombre de stades, de la description de chacun des stades et du mécanisme initiateur du comportement exportateur de la PMI (tableau 1).

TABLEAU 1

Principaux modèles

du processus d'internationalisation de l'entreprise

\begin{tabular}{|c|c|c|}
\hline Auteurs & & Caractéristiques des modèles \\
\hline Bilkey et Tesar (1977) & $\begin{array}{l}\text { Stade 3: } \\
\text { Stade 4: } \\
\text { Stade 5: } \\
\text { Stade 6: }\end{array}$ & $\begin{array}{l}\text { L'entreprise qui n'est pas intéressée par l'export ne répond même } \\
\text { pas à une commande non sollicitée. } \\
\text { Elle est prête à répondre à une commande sollicitée, mais elle ne } \\
\text { fait aucun effort pour explorer la possibilité de développer une } \\
\text { activité d'exportation. } \\
\text { Elle explore activement la possibilité de développer une activité } \\
\text { d'exportation. } \\
\text { Elle exporte sur une base expérimentale vers des marchés } \\
\text { proches psychologiquement. } \\
\text { Elle est une exportatrice confirmée et adapte son niveau } \\
\text { d'exportation de manière optimale. } \\
\text { Elle explore les possibilités de développer une activité d'exportation } \\
\text { vers des pays psychologiquement plus distants. }\end{array}$ \\
\hline Reid $(1981,1983)$ & $\begin{array}{l}\text { Stade 2: } \\
\text { Stade 3: } \\
\text { Stade 4: }\end{array}$ & $\begin{array}{l}\text { Connaissance de l'export - l'entreprise est consciente des } \\
\text { problèmes de reconnaissance d'occasions d'affaires, de } \\
\text { reconnaissance d'un besoin. } \\
\text { Intention d'exporter - il se développe au sein de l'entreprise des } \\
\text { sentiments de motivations et d'attentes à l'égard de l'exportation. } \\
\text { Tentative à l'export - l'entreprise récolte les fruits de son } \\
\text { engagement à l'international. } \\
\text { Acceptation de l'export - au vu des résultats, l'entreprise accepte } \\
\text { ou refuse l'export. }\end{array}$ \\
\hline Cavusgil (1982) & $\begin{array}{l}\text { Stade } 1 \text { : } \\
\text { Stade 2: } \\
\text { Stade 3: } \\
\text { Stade 4: } \\
\text { Stade 5: }\end{array}$ & $\begin{array}{l}\text { Préengagement - l'entreprise n'est présente que sur son marché } \\
\text { domestique et n'est pas intéressée par l'exportation. } \\
\text { Engagement réactif - l'entreprise évalue la possibilité d'exporter } \\
\text { et recherche des informations pour développer cette activité. } \\
\text { Engagement expérimental limité - l'entreprise exporte vers des } \\
\text { pays proches psychologiquement. } \\
\text { Engagement actif - l'entreprise explore systématiquement toutes } \\
\text { les possibilités d'exporter et recourt à la distribution directe. } \\
\text { Engagement intense - l'entreprise partage ses ressources entre } \\
\text { son marché domestique et les marchés étrangers pénétrés. }\end{array}$ \\
\hline
\end{tabular}

Revue internationale P.M.E., vol. 14, $\mathrm{n}^{\circ}$ 2, 2001 
TABleau 1 (suite)

\section{Principaux modèles}

du processus d'internationalisation de l'entreprise

\begin{tabular}{|c|c|c|}
\hline Auteurs & & Caractéristiques des modèles \\
\hline Czinkota (1982) & $\begin{array}{l}\text { Stade } 4 \text { : } \\
\text { Stade } 5 \text { : } \\
\text { Stade } 6 \text { : }\end{array}$ & $\begin{array}{l}\text { L'entreprise est complètement désintéressée par l'export; elle } \\
\text { n'explore même pas la possibilité d'exporter. } \\
\text { L'entreprise est partiellement désintéressée par l'export; elle } \\
\text { explore la possibilité d'exporter qui apparaît comme une activité } \\
\text { incertaine. } \\
\text { L'entreprise est intéressée par l'export ; elle explore activement } \\
\text { les possibilités qu'offre l'exportation et commence à planifier } \\
\text { cette activité. } \\
\text { L'entreprise exporte de façon expérimentale ; son attitude envers } \\
\text { l'export est favorable même si elle n'exploite pas encore toutes } \\
\text { les possibilités qu'offre l'international. } \\
\text { L'entreprise devient une petite exportatrice; son attitude est } \\
\text { favorable et son engagement à l'international, actif. } \\
\text { L'entreprise est une exportatrice expérimentée ; elle a une } \\
\text { attitude très positive à l'égard de l'exportation et planifie tous ces } \\
\text { engagements futurs. }\end{array}$ \\
\hline Moon et Lee (1990) & $\begin{array}{l}\text { Stade } 1: \\
\text { Stade } 2: \\
\text { Stade } 3:\end{array}$ & $\begin{array}{l}\text { Le niveau d'engagement à l'export est faible. } \\
\text { Le niveau à l'international devient modéré. } \\
\text { Le niveau d'engagement sur les marchés étrangers est très } \\
\text { important. }\end{array}$ \\
\hline Crick (1995) & $\begin{array}{l}\text { Stade } 1: \\
\text { Stade } 2: \\
\text { Stade } 3: \\
\text { Stade } 4: \\
\text { Stade } 5: \\
\text { Stade } 6:\end{array}$ & $\begin{array}{l}\text { L'entreprise est complètement désintéressée par l'exportation. } \\
\text { Elle devient partiellement intéressée. } \\
\text { L'entreprise commence à exporter. } \\
\text { L'exportation reste expérimentale. } \\
\text { L'entreprise exporte de façon relativement faible. } \\
\text { L'exportation devient une activité importante au sein de } \\
\text { l'entreprise. }\end{array}$ \\
\hline
\end{tabular}

Source : Adapté de Leonidou et Katsikeas, 1996.

\subsection{Les limites}

Des critiques sur les études concernant le processus d'exportation de la PMI, nous retiendrons deux aspects.

Sur le plan des fondements théoriques, d'une part, les nombreuses études sur le processus d'exportation le considèrent comme une alternative d'expansion du marché domestique, un développement découplé de celui du marché domestique (Kamath et al., 1987). Or, de nombreux cas d'entreprises (Ageron, 1999) montrent la limite de cette affirmation dans la mesure où le partage des ressources d'une PMI exportatrice se fait entre son marché domestique et ses marchés étrangers et, d'autre part, peu d'études s'intéressent à la capacité organisationnelle et industrielle de la PMI dans son comportement exportateur. Comme le soulignent Welch et Luostarinen (1988, p. 37), « le fait de n'étudier que l'activité actuelle sur des marchés étrangers 
occulte un ensemble de changements internes à la PMI. Ces changements sont à la fois le reflet du degré d'internationalisation de la PMI et la conséquence directe du processus d'internationalisation. Ils servent également de bases au développement ultérieur du processus d'internationalisation». On ne saurait alors trop conseiller de recourir à une analyse interne de l'entreprise qui s'apprête à exporter (parce qu'elle le souhaite ou parce qu'elle y est contrainte) pour appréhender de façon globale et pertinente la structuration de son processus d'internationalisation.

Sur le plan méthodologique, les approches sectionnelles croisées ne permettent pas de rendre compte d'un phénomène construit. Les relations causales établies dans ces études ne fournissent aucune compréhension de la dynamique de construction du comportement exportateur d'une entreprise. Ford et Leonidou (1991) insistent par exemple sur le nombre trop limité de critères utilisés dans les classifications mises en évidence. L'utilisation de paramètres de nature statique pour mesurer un phénomène de nature dynamique (Dalli, 1994), le manque de validité du construit et de tests de fiabilité (Moon et Lee, 1990) ont été relevés à plusieurs reprises. En outre, la mesure des variables explicatives manque souvent de sophistication, érigeant ainsi de sérieuses barrières pour une analyse statistique approfondie et une interprétation fine des résultats. L'analyse statistique peut par ailleurs se révéler inadaptée à l'étude d'un phénomène d'une telle complexité, si bien que, pour dépasser les limites inhérentes aux méthodes d'analyse synchroniques, certains auteurs (Debourse et Vasseur, 1985 ; Andersen, 1993) recommandent des études longitudinales; de telles études permettent de réintroduire la dimension séquentielle, évolutive et construite du processus d'exportation.

\section{Le choix du modèle et de la méthodologie}

L'objectif de cet article n'est pas de construire un nouveau modèle du processus d'internationalisation de l'entreprise, mais d'expliquer à partir d'un modèle existant, et en le complétant par de nouvelles variables, la construction du processus d'internationalisation au sein d'une PMI. Nous entendons par capacité de réponse le fait pour un dirigeant d'être simultanément sensibilisé aux stimuli et barrières que constitue la possibilité de marchés à l'international et en mesure de créer le potentiel et les conditions nécessaires à la formulation et à la mise en œuvre d'une stratégie d'internationalisation. Cette sensibilisation ainsi que cette "potentialisation» contribuent à enclencher des processus d'apprentissage qui permettent à l'entreprise de construire et de saisir les possibilités qu' offre l'international. Les modifications ainsi apportées par l'entreprise en termes d'organisation sociale et industrielle caractérisent ces apprentissages. Cette idée de capacité de réponse reste donc complexe dans la mesure où elle concerne les perceptions d'acteurs et, plus particulièrement, du dirigeant face au fait international (en termes d'occasions d'affaires et de contraintes), où elle amène à s'interroger à savoir si les conditions et le potentiel sont suffisants et adaptés pour la formulation et une mise en œuvre

Revue internationale P.M.E., vol. 14, n 2, 2001

(C) 2001 - Presses de l'Université du Québec

Édifice Le Delta I, 2875, boul. Laurier, bureau 450, Sainte-Foy, Québec G1V 2M2 - Tél. : (418) 657-4399 - www.puq.uquebec.ca

Tiré de : Revue internationale P.M.E., vol. $14, \mathrm{n}^{\circ} 2$, sous la direction de Pierre-André Julien. 
internationales ; où elle vise des processus d'apprentissages individuels et organisationnels; où elle participe à des modifications importantes de l'organisation de l'entreprise, de son offre et de son processus de production de même que de l'environnement dans lequel elle évolue.

\subsection{Le choix du modèle}

L'hypothèse implicite de ce travail, hypothèse que nous retenons des études empiriques produites par Ageron et Pellegrin (1996), est qu'on ne peut décrire ni comprendre le processus d'internationalisation sans se référer aux processus de structuration de l'entreprise elle-même. L'ensemble de ces processus de structuration porte aussi bien sur l'organisation structurelle, industrielle que sociale de l'entreprise.

Le modèle fréquemment retenu pour saisir et juger la dynamique du processus d'internationalisation de la PMI reste le modèle développé par Bilkey et Tesar (1977), parce qu'il s'intéresse aux PMI (il a été construit pour ce type d'entreprises, alors que le modèle Uppsala reste plus satisfaisant pour les grandes entreprises) et qu'il a été construit à partir de cas empiriques. Malgré son caractère synthétique, il correspond à la plupart des modèles issus d'études de PME / PMI (Leonidou et Katsikeas, 1996) et souffre de limites importantes (Andersen, 1993; Leonidou et Katsikeas, 1996). Les principales critiques s'articulent autour des quatre points clés suivants :

1. L'étendue des activités considérées : le modèle se rapporte exclusivement au développement des activités exportatrices. Il ignore ainsi les conséquences sur le processus d'internationalisation, d'autres opérations menées au sein de l'entreprise, telles que l'expansion domestique, l'innovation produit, etc. En considérant le processus d'internationalisation comme une alternative d'expansion du marché domestique, un développement découplé de celui des opérations nationales (Kamath et al., 1987), les modèles omettent de souligner que le partage des ressources d'une entreprise, et en particulier de la PMI, s'effectue principalement entre son marché domestique et ses marchés étrangers (Dalli, 1994).

2. Le mode d'engagement sur les marché étrangers : la quasi-totalité des modèles considèrent l'engagement à l'international de l'entreprise comme un processus exclusivement séquentiel. L'hypothèse centrale est celle d'un développement international fondé sur une série de décisions incrémentales. Le caractère linéaire et ordonné de la démarche laisse dès lors supposer que l'engagement à l'international est mécaniquement lié aux dotations de ressources croissantes pour l'exportation et aux opportunités de marché (Julls et Waters, 1987). Or, la linéarité du processus ignore le 
raccourcissement des étapes pour des entreprises disposant d'une assez forte maturité internationale (Millington et Bayliss, 1990). À l'inverse, le caractère parfois statique et l'inertie du processus sont fréquemment observés lors des premières phases d'exportation (en témoigne le cas étudié), tout comme les désinvestissements ou autres engagements des marchés étrangers.

3. La dynamique et la vitesse de développement international : bien qu'il soit reconnu que le processus d'exportation est profondément dynamique et doté d'une forte temporalité, la plupart des modèles apparaissent statiques et décontextualisés. La théorie ne met pas en évidence des paramètres importants comme le type de pays accueillant les investissements, le nombre de clients concernés et les canaux utilisés pour entrer sur les marchés étrangers. Les variables affectant le passage d'un stade à un autre sont systématiquement occultées et la vitesse de cheminement au sein des étapes n'est, si parfois expliquée, jamais considérée. Le modèle de Bilkey et Tesar focalise principalement sur les caractéristiques du marché et sur les caractéristiques managériales de la petite entreprise pour juger du processus d'internationalisation. Or, on sait que l'intensité technologique, le cycle de vie du produit (Young, 1987) et la structure organisationnelle (Kalika, 1988) peuvent affecter la vitesse de progression de la firme tout au long de sa trajectoire internationale.

Cette dernière limite nous conduit à préférer un cadre beaucoup plus intégrateur qui met en évidence les variables clés de la décision d'exporter. Comme le propose Gibiat (1994), retenons les trois variables explicatives suivantes de la décision d'exporter:

- les caractéristiques de l'environnement, qui nous renvoient aux notions d'appartenance sectorielle, d'accès aux ressources, de type de clientèle, d'avantage concurrentiel, etc. ;

- les caractéristiques de l'entreprise, soit la structure organisationnelle, les caractéristiques du processus de production, les caractéristiques des produits, etc. ;

- les caractéristiques du dirigeant, soit son expérience, son attitude à l'égard de l'export, etc.

\subsection{La méthodologie de recherche}

\subsubsection{Les choix méthodologiques}

Les limites évoquées précédemment justifient le choix d'une approche longitudinale afin que nous puissions introduire une dimension temporelle dynamique dans l'étude du processus d'exportation :

Revue internationale P.M.E., vol. 14, n 2, 2001

(C) 2001 - Presses de l'Université du Québec

Édifice Le Delta I, 2875, boul. Laurier, bureau 450, Sainte-Foy, Québec G1V 2M2 • Tél. : (418) 657-4399 - www.puq.uquebec.ca

Tiré de : Revue internationale P.M.E., vol. 14, $\mathrm{n}^{\circ} 2$, sous la direction de Pierre-André Julien. 
La plupart des travaux sur l'internationalisation s'appuient sur des méthodologies d'analyse synchronique [...] Ils étudient un échantillon de firmes à un moment déterminé dans le temps. La seconde approche, plus difficile à mettre en œuvre, à savoir l'approche diachronique de l'évolution d'une entreprise face au fait international, mérite d'être mise en œuvre. L'internationalisation est un processus qui se déroule dans le temps ; l'étudier peut rendre opportune l'introduction du temps dans l'analyse. Ceci doit permettre de mieux comprendre comment s'effectue dans une entreprise l'interaction entre son évolution internationale et les autres caractéristiques de son évolution et comment jouent de façon précise les déterminants; de mieux mesurer les modifications de l'organisation et ainsi de suite. (Debourse et Vasseur, 1985, p. 8)

Notre ambition est d'expliquer la construction du processus d'internationalisation d'une PMI à travers les différents stades qu'elle traverse au cours de son engagement sur des marchés étrangers. L'analyse d'un tel phénomène (la construction d'une capacité de réponse) renvoie à trois méthodologies (Yin, 1994) : la méthode historique, l'étude de cas et l'expérimentation. La principale distinction entre ces trois stratégies est la suivante: la méthode historique traite d'un passé «mort» comme, par exemple, le cas où aucune personne pertinente pour l'objet de la recherche n'est plus en vie pour rapporter, même rétrospectivement, ce qui s'est passé ; ce manque conduit l'investigateur à utiliser des archives, etc. À l'inverse, l'étude de cas permet, par l'intermédiaire d'entretiens et d'observations directes, de rendre compte de ce qui s'est passé, même si l'investigateur n'a pas la possibilité de manipuler ni d'influencer directement et consciemment l'objet étudié. Enfin, l'expérimentation permet à l'investigateur de manipuler le déroulement de l'action en isolant et modifiant une ou plusieurs variables pour constater l'incidence de la modification dans l'expérience.

Si l'étude de cas nous permet de répondre à la question du «comment» et d'appréhender le processus d'internationalisation de façon dynamique, elle ne doit pas tomber dans les travers relevés par Bilkey et Tesar (1977) pour expliquer les passages successifs du modèle, à savoir le nombre trop élevé de variables qui influencent le comportement exportateur des PMI. Pour que l'étude de cas soit pertinente, il est nécessaire d'introduire un éclairage particulier qui nous permette de comprendre et d'analyser le processus dynamique et complexe d'internationalisation. L'histoire générale de l'entreprise nous pousse une fois de plus à considérer le produit comme l'élément central de cette construction. Nous rejoignons ainsi Lanzara (1987) qui, au cours d'une analyse menée sur les facteurs qui ont permis aux PMI italiennes d'atteindre et de maintenir une position compétitive sur les marchés étrangers, arrive à la conclusion suivante :

[...] la manière dont les entreprises et, plus particulièrement, les petites, gèrent leur système de production et leur technologie est cruciale pour leur succès à l'export. Ceci suppose que les explications sur le processus d'expansion sur des marchés étrangers sont incomplètes à moins que l'on prête une attention spécifique à l'environnement opérationnel qui conduit à une telle expansion. (Lanzara, 1987, p. 43)

Revue internationale P.M.E., vol. 14, $\mathrm{n}^{\circ}$ 2, 2001 


\subsubsection{La démarche méthodologique}

La monographie présentée s'inscrit dans un projet de recherche au sein de l'ESA CNRS 5055, avec la région Rhône-Alpes sur le thème : «Les conditions préalables au sein des PMI rhônalpines, aux processus de modernisation et internationalisation : cohérence entre logiques sociales et logiques industrielles ». Elle s'appuie sur une étude réalisée auprès d'une PMI exportatrice, qui a été choisie parce qu'elle répondait aux conditions suivantes : avoir moins de 500 salariés, être totalement indépendante et produire un bien manufacturier.

Pour l'étude de cette PMI, voici quelle a été la démarche de recherche:

- un premier entretien téléphonique avec le dirigeant de l'entreprise pour la préparation de la collecte des données. Nous lui avons présenté de façon succincte la recherche, ses objectifs, nos motivations et avons cherché à connaître ses intentions, ses attentes, ses interrogations ;

- un deuxième entretien ${ }^{5}$ a eu lieu avec le PDG. Cet entretien, déterminant pour la suite de la recherche, nous a permis de saisir l'histoire de l'entreprise (trajectoire des activités domestiques et internationales). De la création de la PMI à sa situation actuelle, il nous a permis, dans un premier temps, de cerner les moments importants de l'entreprise et, à partir de ces moments clés, de déterminer ses différentes phases de développement. Une fois traité, cet entretien nous a fourni une trame générale, base pour un second entretien avec le dirigeant ;

- lors du troisième entretien, des points importants, obscurs ou non compris ont été précisés. Un peu plus directif, il nous a permis d'analyser plus finement chacune des phases définies lors du traitement du précédent entretien. Nous avons commencé à saisir et à comprendre les articulations qui se sont mises en place tout au long du développement de l'entreprise entre la stratégie globale, la stratégie industrielle et l'activité d'exportation;

- un quatrième entretien avec le responsable de production et un quatrième avec le responsable des achats nous ont permis de cerner l'organisation industrielle et sociale en place. Beaucoup plus techniques, ces entretiens ont nécessité, de notre part, une grande vigilance pour éviter tout oubli d'informations ;

- un cinquième entretien avec le responsable de l'agence de distribution. Cette personne est à l'emploi de l'entreprise depuis sa création.

5. Notons que nous avons également réalisé deux entretiens téléphoniques supplémentaires : un premier avec le responsable financier et un second avec le dirigeant de l'entreprise.

Revue internationale P.M.E., vol. 14, n 2, 2001

(C) 2001 - Presses de l'Université du Québec

Édifice Le Delta I, 2875, boul. Laurier, bureau 450, Sainte-Foy, Québec G1V 2M2 • Tél. : (418) 657-4399 - www.puq.uquebec.ca

Tiré de : Revue internationale P.M.E., vol. $14, \mathrm{n}^{\circ} 2$, sous la direction de Pierre-André Julien. 
Ces sept entretiens, d'une durée moyenne de quatre heures (à l'exception des entretiens téléphoniques nécessairement plus courts), nous ont permis de rédiger deux documents d'analyse :

- une monographie de l'entreprise qui constitue la représentation, en trajectoires, de l'histoire de l'entreprise ;

- une matrice (construits, temps) construite à partir de la technique «time ordered matrix » (Huberman et Miles, 1991) et qui consiste à codifier les données.

\section{Les principaux résultats exploratoires}

L'histoire de l'entreprise commence à la fin des années 1950, le dirigeant, ingénieur de formation, doté d'un savoir-faire important, tant au niveau de l'électronique que de la mécanique, cherche à développer des automatismes : il crée la société $\mathrm{X}$. Son projet initial n'est pas la création de produits mais la résolution de problèmes : répondre à une demande spécifique d'un client, concevoir une solution, développer le produit correspondant et mettre ainsi ses connaissances à la disposition d'autres entreprises.

Le dirigeant commence alors à développer ses propres produits (le boîtier électronique, l'alarme) et dépose de nombreux brevets en son nom propre. Le développement de l'activité de l'entreprise conduit le dirigeant à investir (achats d'outillages et de véhicules) et à embaucher des ouvriers, en fait des « compagnons » selon les termes du dirigeant fondateur, qui emploie des amis plus que des personnes choisies spécialement pour leur qualification.

C'est à cette époque que l'entreprise décide d'orienter son activité vers le contrôle d'accès et la gestion des ouvertures, mission qui, dans l'esprit du dirigeant, prend la forme de l'installation et de la mise en service de produits conçus et fabriqués dans l'entreprise. La région d'implantation de l'entreprise va jouer le rôle de catalyseur. L'entreprise saisit ce nouveau créneau en concevant un nouvel automatisme de portail : l'automatisme hydraulique utilisant pompe, vérins, huile, etc. À partir de ce moment-là, selon les termes de son dirigeant actuel, «l'entreprise a pris son sens».

En 1965, l'entreprise se développe : une équipe de cinq à six personnes travaille à temps plein sur la fabrication et l'installation de portails qui deviennent une préoccupation importante de l'activité de l'entreprise. Un événement va orienter le dirigeant fondateur vers la porte automatique piétonne : un ami miroitier a obtenu un gros marché et lui demande de fabriquer et d'installer des portes automatiques. L'installation et l'utilisation des portes sont difficiles (problème de fiabilité), mais la porte automatique en elle-même est un succès. L'année suivante, 10 portes piétonnes 
sont fabriquées et installées et le brevet de la porte automatique piétonne est déposé en 1967. L'activité liée à l'installation des alarmes est peu à peu abandonnée au profit de l'activité de conception de portes automatiques qui se développe.

En 1977, le dirigeant, qui sent qu'il faut développer encore plus cette activité de portes automatiques qui est un créneau porteur, ne veut pas le faire tout seul. Il demande de l'aide à ses frères qui viennent en France (l'un travaille en Suisse comme directeur d'ateliers, l'autre en Italie comme ingénieur). Ensemble, ils créent la SARL X. L'entreprise se structure et chaque frère se spécialise dans un domaine d'activité : l'un à la conception, un autre aux études et le troisième à la production. La standardisation du produit est recherchée, la production sur catalogue s'ajoute à la fabrication sur mesure. Toutefois, le marché régional trop petit pour le développement de l'activité de l'entreprise la conduit à se déplacer sur le marché national, où la concurrence est faible à cette époque.

Au début des années 1980, un événement fait basculer définitivement l'activité de l'entreprise vers la porte automatique : la rencontre avec une société agenceur d'hôpitaux qui, en réponse à un appel d'offres, sollicite l'entreprise X pour la conception de systèmes d'ouverture de portes. Ce système d'ouverture permet à l'entreprise X de se lancer sur un créneau en pleine expansion (secteur hospitalier). Cette demande va provoquer un nouveau dynamisme chez l'entreprise X. De nombreux modèles de portes sont déposés et une gamme de produits se crée. L'entreprise offre, dès lors, un catalogue de portes dont les principales variables sont la longueur et la couleur.

En 1983, le fils du dirigeant fondateur reprend l'entreprise avec l'un de ses oncles. La technologie utilisée est une technologie maîtrisée. Le système industriel et logistique se développe. La standardisation du produit et la régularité des flux de commandes (50 à 100 portes par mois) permettent la production en série à des prix intéressants, la production à la commande restant cependant prépondérante. Comme une partie des composants est maintenant achetée à l'extérieur, l'assemblage et l'installation prennent le pas sur la fabrication. Le personnel, devenu polyvalent, est employé indifféremment à la fabrication, à l'assemblage, à l'installation ou au dépannage. Le dépannage devient d'ailleurs une nouvelle donnée pour l'entreprise $X$ : il crée une relation de confiance avec le client et permet à la SARL X de se différencier des autres entreprises en créant son propre réseau de dépannage, offrant ainsi un service rapide et de qualité qui favorise la fidélisation.

Dès 1984, Philippe Réolon réorganise la SARL X. Son intention délibérée est de déléguer: «Un chef d'entreprise ne doit pas s'occuper de tout, il doit développer simplement le produit à installer demain. Il doit guider l'entreprise dans la trajectoire qu'il a choisie. Il est en fait le gardien de la bonne conduite de l'entreprise. » Cette prise de conscience aboutit à la création, en parallèle de la SA X, d'une

Revue internationale P.M.E., vol. 14, n 2, 2001

(C) 2001 - Presses de l'Université du Québec

Édifice Le Delta I, 2875, boul. Laurier, bureau 450, Sainte-Foy, Québec G1V 2M2 • Tél. : (418) 657-4399 - www.puq.uquebec.ca

Tiré de : Revue internationale P.M.E., vol. 14, $\mathrm{n}^{\circ} 2$, sous la direction de Pierre-André Julien. 
seconde société dont l'activité principale sera l'installation (l'activité de la SA X est la fabrication). Deux métiers différents sont ainsi mis au jour: un métier de fabricant et un métier d'installateur.

L'entreprise, qui jouit d'une renommée nationale considérable, se lance à la recherche de nouveaux marchés. Le succès obtenu au salon BATIMAT 1993 (médaillée du concours de l'innovation) constitue à la fois un signe de la qualité de la nouvelle organisation et un tremplin pour le développement de l'entreprise à l'international.

L'histoire de l'entreprise nous permet de mettre en évidence une trajectoire d'ensemble de l'internationalisation. La PMI, qui n'exporte pas du tout (stade 1) au début de sa vie, va peu à peu réaliser des coups à l'international par l'intermédiaire d'autres entreprises (stade 2). De plus en plus sollicitée, elle va chercher à développer cette activité à l'international (stade 3) et à établir une véritable stratégie à l'international.

Stade 1. La PMI n'est pas intéressée par l'exportation ; elle ne répond même pas à une commande non sollicitée.

Au sein de l'entreprise, ce stade 1 est caractérisé par un dirigeant fondateur qui, par son esprit créateur et son savoir-faire important, tant au niveau électronique qu'au niveau mécanique, cherche à développer des automatismes. L'entreprise $\mathrm{X}$ est créée. Développer des produits à la demande devient le métier du dirigeant. Tous les produits conceptualisés sont ensuite développés par le chef d'entreprise. À travers cette créativité, l'entreprise $\mathbf{X}$ acquiert une renommée au niveau régional par la simplicité et la «débrouillardise» de son dirigeant.

L'activité de chantier commence réellement à naître et nécessite l'achat d'outillage, de véhicules, ainsi que l'embauche d'ouvriers. L'idée de compagnon est très importante dans la tête du dirigeant qui emploie des amis plus que des personnes qualifiées pour un certain type de travail.

La région d'implantation de l'entreprise a été un facteur de succès pour l'entreprise X; il s'agit d'une ville riche où la demande en portail est importante. Le dirigeant souhaite occuper ce nouveau créneau en développant des automatismes de portail. Pour ce faire, un nouvel automatisme est conçu : un automatisme hydraulique (pompe, vérins, huile).

L'entreprise se développe et le dirigeant crée les Ets X. Une équipe de cinq à six personnes qui travaille à temps plein sur la fabrication et l'installation de portails. Cette équipe, principalement composée de proches du père, possède peu de savoir-faire en matière d'automatisme. Mais le dirigeant est un concepteur très averti qui dépose de nombreux brevets : brevets d'alarmes, dispositifs de vérins, motorisation de portails et système d'ouverture par électrovanne. 
Un événement va orienter le dirigeant vers la porte automatique piétonne : un ami miroitier a obtenu un gros marché et lui demande de fabriquer et d'installer des portes automatiques. L'installation et l'utilisation des portes sont difficiles (problèmes de fiabilité), mais la porte en elle-même est un succès. L'année suivante, 10 portes piétonnes sont fabriquées et installées. Le brevet de la porte automatique piétonne est déposé en 1967, les autres activités sont peu à peu abandonnées.

\section{Le passage du stade 1 au stade 2}

Le dirigeant sent qu'il faut développer encore plus cette activité qui est un créneau porteur, mais il ne veut pas le faire tout seul. Il demande donc de l'aide à ses frères; ensemble, ils créent la SARL X. L'entreprise se structure : chaque frère se spécialise dans un domaine d'activité (conception, études et production). La standardisation du produit est recherchée et la production sur catalogue s'ajoute à la fabrication sur mesure. L'entreprise cherche toujours à employer les nouvelles technologies pour l'ouverture de portes. La pneumatique est utilisée pour le développement de nouveaux systèmes d'ouverture et remplace la motorisation par vérins hydrauliques.

Simultanément, l'entreprise commence à répondre à des marchés étrangers de façon très sporadique et non organisée : l'internationalisation correspond à des opérations de prestige toujours en sous-traitance. Selon l'expression du dirigeant, «l'entreprise fait des coups à l'international ». Le scénario suivi pour toutes ces actions à l'international est identique : une société française qui connaissait la SARL X et qui travaillait déjà avec l'entreprise sur le marché régional décrochait un marché / chantier pour une haute personnalité ou une famille royale. Cette société, qui devait traiter le marché de A à Z, s'occupait de trouver les soustraitants nécessaires au bon déroulement des travaux. Pour les portes automatiques, l'entreprise X obtenait toujours le marché. Ce type de marchés à l'étranger était monopolisé par quelques entreprises qui avaient déjà réalisé des opérations de prestige et qui obtenaient toujours ces chantiers très spécifiques. Un effet de boule de neige s'était créé : une réalisation pour le roi du Gabon entraînait une réalisation pour le roi du Maroc, et ainsi de suite. De ces chantiers, on retiendra deux caractéristiques principales :

- l'entreprise $X$ ne fait aucune démarche pour obtenir ces chantiers : elle est prospectée et retenue pour la qualité et l'ingéniosité de la conception de ses produits. C'est la reconnaissance régionale qui permet à l'entreprise $\mathrm{X}$ de décrocher ces chantiers ;

- les produits vendus sont toujours des produits spécifiques, produits sur mesure qui représentent des opérations de prestige. 
L'absence de démarche active de la part du dirigeant, malgré une personnalité innovante et curieuse, tient au fait que la demande potentielle du marché régional et national apparaît suffisante pour assurer et soutenir le développement futur de l'entreprise. On peut également noter que le produit, qui reste sur mesure, ne peut être exporté, car, selon le dirigeant: «on ne peut concevoir son activité hors de son marché national, quand on ne maîtrise pas complètement le produit que l'on propose ». Cela explique que le dirigeant ne s'engage pas à l'international et que, par conséquent, ce soit le donneur d'ordres lui-même qui prend en charge ce processus d'exportation.

Stade 2. La PMI est prête à répondre à une commande non sollicitée, mais elle ne fait aucun effort pour explorer la possibilité de développer une activité d'exportation. Simultanément à ces quelques coups à l'international, la société se déplace d'un marché régional sur le marché national, marché peu concurrencé à cette époque.

Un événement fait basculer définitivement l'activité de l'entreprise vers la porte automatique : la rencontre avec une société (société agenceur d'hôpital) qui, en réponse à un appel d'offres d'un hôpital lyonnais, sollicite l'entreprise X pour la conception de systèmes d'ouverture de portes. Ce système d'ouverture offre à l'entreprise X une niche peu concurrencée sur un marché en expansion, celui du secteur hospitalier dans une région où l'hôpital tient une place importante. Cette demande va provoquer un nouveau dynamisme. Le milieu hospitalier permet un développement massif de la porte automatique et il correspond à $80 \%$ de la production de l'entreprise à cette époque. Il faut, en effet, suivant les demandes et les possibilités des bâtiments, installer et, si nécessaire, créer de nouvelles portes. De nombreux modèles de portes et une gamme de produits apparaissent : portes en V, portes en panneaux coulissants, portes battantes, etc. L'entreprise X offre dès lors un catalogue de portes dont les principales variables sont la longueur et la couleur. Cela permet de satisfaire plus facilement la demande : on coupe à la longueur souhaitée et on assemble tous les éléments.

Après le décès de son père, le dirigeant actuel reprend l'entreprise avec l'un de ses oncles. La technologie utilisée est une technologie maîtrisée. Le système industriel et logistique se développe. La standardisation du produit et la régularité des flux de commandes (50 à 100 portes par mois) permettent la production en série à des prix intéressants, la production à la commande restant cependant prépondérante. Comme une partie des composants est maintenant achetée à l'extérieur, l'assemblage et l'installation prennent le pas sur la fabrication. Le personnel devenu polyvalent est employé indifféremment à la fabrication, à l'assemblage, à l'installation ou au dépannage. Le dépannage devient d'ailleurs une nouvelle donnée pour l'entreprise : cette activité crée une relation de confiance avec le client et permet à l'entreprise X de se différencier des 
autres entreprises en créant son propre réseau de dépannage offrant ainsi un service rapide et de qualité favorisant sa fidélisation. De nouveaux modèles se créent toujours en tenant compte de l'installation qui en résulte ainsi que du service aprèsvente. «Le produit en soi ne doit pas être une simple porte que l'on installe chez le client sans se soucier de ce qu'il devient après, mais un bien qu'il faut chérir et surveiller », rappelle t-on déjà dans l'entreprise.

\section{Le passage du stade 2 au stade 3}

Le dirigeant actuel réorganise la SARL X. Son intention délibérée est de déléguer : «Un chef d'entreprise ne doit pas s'occuper de tout, il doit développer simplement le produit à installer demain. Il doit guider l'entreprise dans la trajectoire qu'il a choisie. Il est en fait le gardien de la bonne conduite de l'entreprise. » Les mutations du monde de l'entreprise des années 1980, le nouveau dirigeant les comprend bien : mieux définir le métier en concentrant l'activité de l'entreprise sur les études et le montage ; réorganiser en conséquence en créant le bureau d'étude (dont il est au départ le seul employé) ou en mettant en place un système d'information de façon à mieux cerner le coût des produits ; maintenir la culture du fondateur en déléguant, en favorisant la circulation de l'information et la responsabilité.

Dès lors, l'entreprise cherche à développer son activité à l'international et commence à exporter vers d'autres pays de la CEE. La prise de conscience des possibilités qu'offrent les marchés étrangers devient de plus en plus forte au sein de l'entreprise. On peut d'ailleurs noter que si cette prise de conscience ne s'est pas traduite dans un premier temps au niveau de la démarche d'exportation, elle trouve sa concrétisation dans la recherche d'une standardisation accrue du produit. Les quelques coups à l'international de l'entreprise montrent au dirigeant actuel que la recherche d'activité sur des marchés étrangers constitue un vecteur de développement essentiel pour l'entreprise.

Deux événements représentent, dans l'esprit du dirigeant actuel, l'amorce du développement à l'international de la société. Le premier, à l'époque de la SARL X, n'est pas, à proprement parler, une expérience internationale puisqu'elle concerne une affaire à la Réunion : un client de la Réunion demande à être le concessionnaire de l'entreprise X sur l'île. Ainsi, pour la première fois, loin de sa base, $\mathbf{X}$ est amenée à vendre un produit non plus spécifique, mais issu de son catalogue. Activité née d'une concession, conduisant à la vente de produits standards, cette expérience se renouvellera, en 1993, avec l'agence de Mulhouse qui obtiendra quelques chantiers en Allemagne. Ainsi, pour la première fois, même si l'export n'a pas été recherché en tant que tel, $X$ est à l'origine et à la réalisation, grâce à ses deux entités, d'une activité à l'international qui devient de plus en plus importante.

Revue internationale P.M.E., vol. 14, nº 2, 2001

(C) 2001 - Presses de l'Université du Québec

Édifice Le Delta I, 2875, boul. Laurier, bureau 450, Sainte-Foy, Québec G1V 2M2 • Tél. : (418) 657-4399 - www.puq.uquebec.ca

Tiré de : Revue internationale P.M.E., vol. $14, \mathrm{n}^{\circ} 2$, sous la direction de Pierre-André Julien. 
Stade 3. La PMI explore activement la possibilité de développer une activité d'exportation; elle exporte sur une base expérimentale vers des marchés psychologiquement proches.

L'année 1989 constitue un tournant dans le développement de l'entreprise. Le dirigeant actuel crée en parallèle de la SARL une seconde société, la SA X. La SARL va s'occuper de l'activité de fabrication, mais les activités de conception et d'assemblage seront transférées au sein de la SA X. Cette restructuration de l'entreprise met au jour deux métiers complètement différents : un métier de fabricant et un métier d'installateur.

La SA X, en charge de la fabrication de la porte automatique qui a fait sa renommée nationale, affirme la vocation internationale de l'entreprise à la recherche de nouveaux marchés. Le succès obtenu au salon BATIMAT 1993 (médaillée du concours de l'innovation) est à la fois un signe de la qualité de la nouvelle organisation et un tremplin pour le développement de l'entreprise à l'international.

Dans le même temps, la cassure entre les activités de fabrication et d'installation est réalisée par la création de l'AGENCE X et le développement d'un réseau de 20 concessionnaires.

Peu à peu, la fabrication va être transférée de la SARL vers la SA X, l'activité de fabrication s'efface (sauf pour les prototypes) au profit de la sous-traitance et, en 1994, la SARL disparaît juridiquement. Dans le même temps, la société AGENCE $\mathrm{X}$ crée trois nouvelles agences (Bretagne, Paris, Alsace) chargées d'accroître l'implantation des activités de distribution, d'installation et de dépannage.

Cette restructuration permet au dirigeant de changer sa politique à l'international. $L$ 'entreprise a la volonté de développer son activité vers l'export. Elle cherche «à trouver des partenaires », comme le souligne le dirigeant actuel. Mais la relation $\mathrm{X}$ - distribution à l'export sera différente de la relation $\mathrm{X}$ distribution en France. Les partenaires étrangers ne seront pas de simples distributeurs et installateurs de portes automatiques, ils devront en plus de ces deux fonctions fabriquer les portes. À ce stade, la fabrication signifie stockages de matières premières et de composants (radars, cellules), de caissons filé-aluminium qui seront ensuite découpés à la longueur souhaitée et peints de la couleur demandée. Les distributeurs étrangers deviennent ainsi des fabricants de produits conçus par X, produits qu'ils adaptent aux spécificités de la demande. Contrairement à ce qui se passe avec les concessions en France, $X$ n'est plus le fabricant, mais le partenaire : des délais commande-livraison réduits et une proximité fabricant-client assurent une meilleure satisfaction. Pour le dirigeant, ce désir d'aller à l'export a été réalisé grâce à l'industrialisation complète du produit. «Jusqu'en 1993, insiste-t-il, de nombreux composants étaient déjà standardisés, mais la porte 
ne l'était pas complètement. Le passage du mécano-soudé au filé-aluminium a facilité la standardisation des caissons et a permis, en même temps, la standardisation du produit. Les caissons filé-aluminium ont ainsi pu être stockés à des longueurs standards pour être par la suite coupés à la longueur commandée, alors que précédemment chaque commande engendrait la fabrication d'un caisson spécifique. » Ce passage à l'industrialisation rejette tout ce qui est spécifique. «L'entreprise, ajoute-t-il, ne veut fabriquer que du standard. Ce qui intéresse les clients, c'est qu'on leur propose un catalogue de produits et nous avons su intégrer les remarques des clients pour notre développement à l'export. Nombreux étaient, en effet, ceux qui nous disaient : le jour où votre produit sera industrialisé, revenez nous voir, car ce produit nous intéresse mais, pour l'instant, vous ne vendez que du spécifique et c'est impossible pour nous. »

Cette industrialisation débouche dès lors sur l'intégration des fournisseurs, intégration qui devient le pilier d'une stratégie industrielle internationale. Cette stratégie repose sur une conception de base centralisée dans le pays d'origine; une déconcentration d'une partie de l'activité de fabrication pour être plus proche du marché ; une activité d'assemblage faite à proximité du marché assurant ainsi une meilleure adaptation du produit au besoin du client ; enfin, un service (installation et après-vente) internationalisé.

L'histoire de l'entreprise et de son processus d'internationalisation est résumée au tableau 2 .

Remarquons que les éléments que nous proposons dans les colonnes ne sont pas sans rappeler ceux évoqués dans la plupart des modèles de la décision d'exporter. Nous avons ainsi caractérisé la qualité et le dynamisme du management à travers l'organisation sociale introduite par le dirigeant par l'intermédiaire des systèmes d'animation (Bilkey et Tesar, 1977 ; Cavusgil, 1982). Par ailleurs, la considération de l'organisation industrielle rend compte des barrières et des stimuli mis en évidence dans ces mêmes modèles (Wortzel et Wortzel, 1981 ; Crick, 1995). En effet, ces auteurs insistent fréquemment sur les rôles que peuvent jouer le produit (notamment en termes de différenciation) ainsi que la distribution (trouver un système adéquat) dans le processus d'exportation de la PMI.

\section{Enseignements et implications}

À partir de ce cas empirique, il nous semble important de revenir sur ses deux éléments saillants : d'une part, nous remarquons que la logique de standardisation constitue l'élément majeur, le vecteur de l'internationalisation. En effet, le fait pour l'entreprise de proposer un produit standard lui permet de s'engager de façon active à l'international. D'autre part, la cohérence entre l'offre, le processus de production et l'organisation industrielle semble être un élément essentiel du processus de

Revue internationale P.M.E., vol. 14, n 2, 2001

(C) 2001 - Presses de l'Université du Québec

Édifice Le Delta I, 2875, boul. Laurier, bureau 450, Sainte-Foy, Québec G1V 2M2 - Tél. : (418) 657-4399 - www.puq.uquebec.ca

Tiré de : Revue internationale P.M.E., vol. $14, \mathrm{n}^{\circ} 2$, sous la direction de Pierre-André Julien. 


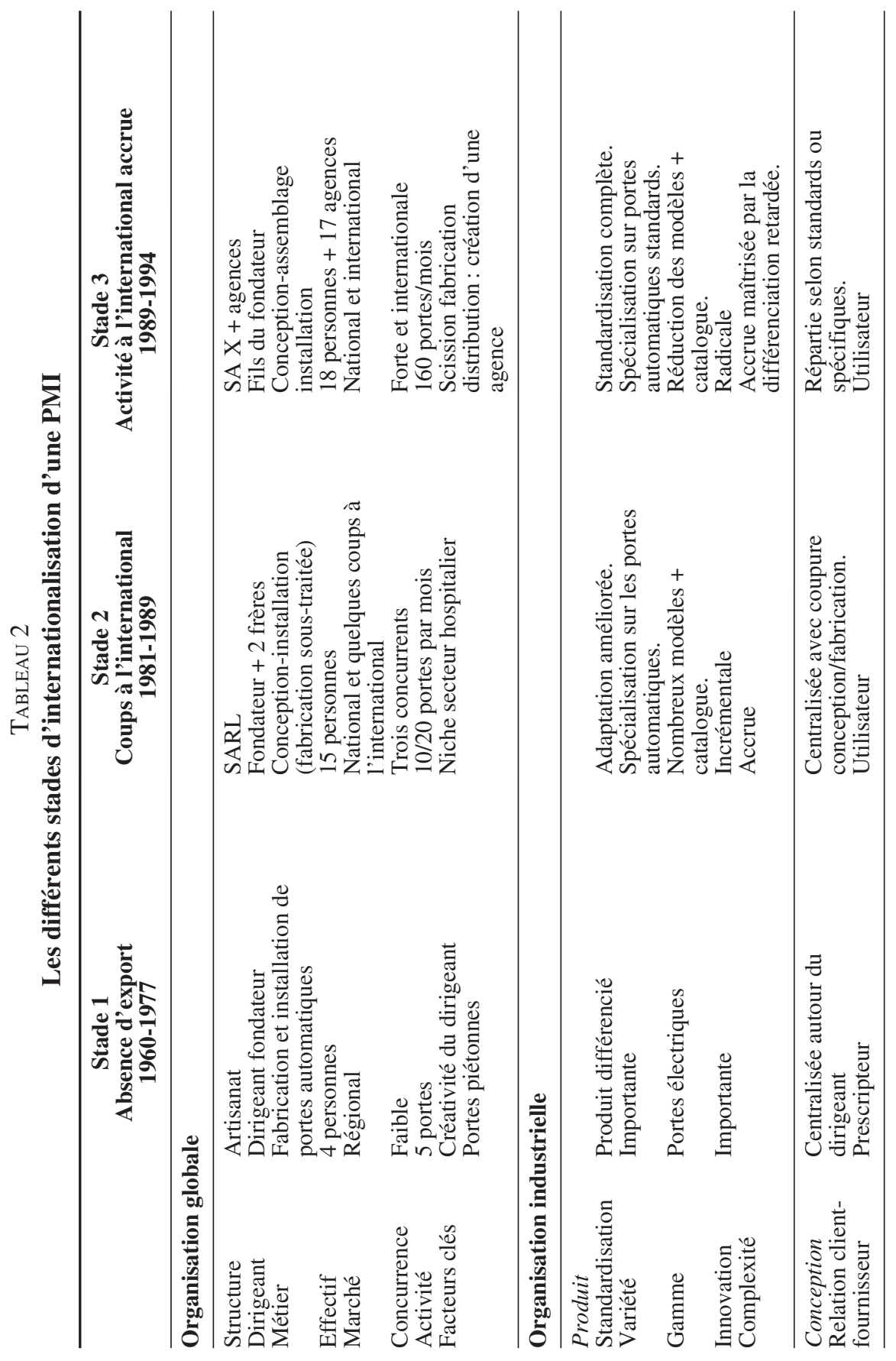

Revue internationale P.M.E., vol. 14, $\mathrm{n}^{\circ}$ 2, 2001 


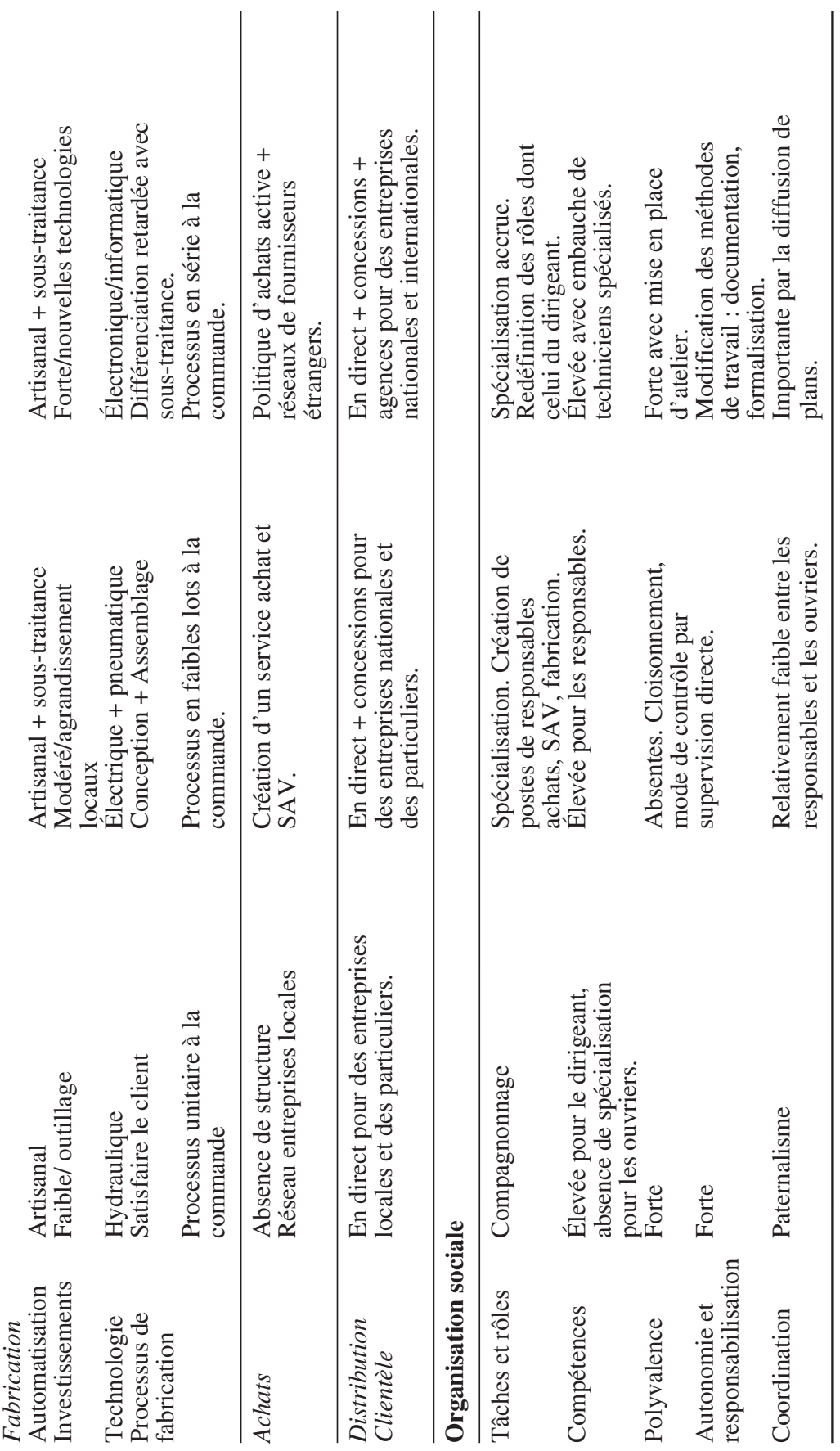

Revue internationale P.M.E., vol. 14, $\mathrm{n}^{\circ}$ 2, 2001 
structuration de l'entreprise et, donc, de son engagement sur des marchés étrangers. En ce sens, nous rejoignons les travaux empiriques d'INTERSTRATOS (1995) et de ALGOE (1993) qui insistent sur le rôle joué par cette cohérence dans le processus d'internationalisation de la PME/ PMI.

Plus généralement, les conclusions auxquelles le cas nous permet d'aboutir à propos de la décision d'exporter et de la structuration du processus d'internationalisation peuvent être explicitées de la façon suivante:

- l'importance relative de certains facteurs par rapport à d'autres apparaît nettement dans le cas étudié. Ainsi, il ressort que, selon les stades d'internationalisation, l'effort de l'entreprise ne porte pas sur les mêmes éléments, comme le montre le tableau suivant (tableau 3 ).

Les variables structurantes du processus d'internationalisation changent selon le stade d'internationalisation : elles sont plus ou moins importantes, constituent des facteurs clés ou secondaires du développement international et évoluent différemment au cours des stades d'internationalisation (un facteur clé à un stade peut devenir secondaire à un autre stade). En ce sens, notre recherche montre que la phase de structuration d'une variable est fréquemment suivie d'une phase d'accommodation au cours de laquelle l'entreprise doit mettre en adéquation l'ensemble de ses autres variables pour favoriser la réussite de son développement futur.

Le processus d'exportation, beaucoup plus qu'une recherche de nouveaux territoires, repose fondamentalement sur une recherche de cohérences. En effet, comme cela a été montré par ailleurs, les démarches d'internationalisation ont en commun la capacité des entreprises à mettre en œuvre des cohérences entre un ensemble de variables fondamentales du questionnement international, plus particulièrement du système industriel, comme en témoigne le cas X. La variable international ne peut, par conséquent, se réduire à la seule variable marché, et implique également une prise de position de l'entreprise en termes de produits, de modalités d'entrée et de compétences développées. Cela peut expliquer que certaines petites entreprises sont d'emblée à l'international, alors que d'autres témoignent d'un processus d'internationalisation beaucoup plus long et séquentiel (local, national et international), voire de processus de dés-internationalisation caractérisés par des reculs, des bifurcations, voire des échecs sur certains marchés étrangers.

Les questionnements soulevés et posés par l'exportation doivent s'inscrire dans une démarche stratégique générale, en ce qu'il questionne les variables traditionnelles du raisonnement stratégique (le produit, l'organisation, l'environnement concurrentiel, etc.). En cela, nous rejoignons les travaux de Reid (1981), Reid et Rosson (1982) et Julien et al. (1995) qui affirment que pour « les PME, l'exportation ne se dissocie pas de l'ensemble des activités et notamment de la vision globale du dirigeant. Plusieurs recherches semblent avoir été menées dans une perspective laissant supposer que l'exportation doit relever d'une fonction bien distincte au sein 
de l'entreprise [...] Or, il semble que ce soit une erreur d'isoler les activités d'exportation de la dynamique d'évolution de l'entreprise » (Julien, Léo et Philippe, 1995, p. 60).

Le dirigeant est un élément fondamental du processus décisionnel à l'international. À cet égard, le cas étudié est riche d'enseignements, notamment en ce qu'il permet de saisir l'attitude du dirigeant tout au long du processus d'internationalisation; en outre, il montre que l'image du dirigeant opportuniste agissant essentiellement selon son intuition plutôt qu'en suivant une méthode structurée et planifiée s'estompe au profit de comportements visionnaires planificateurs et stratégiques, au fur et à mesure que le développement à l'international s'intensifie. Ainsi, à travers ce cas, on retrouve les quatre phases du processus de décision d'exporter mis en évidence par Gibiat (1994, p. 21):

- une phase de prise de conscience «qu'une stratégie d'exportation peut être considérée comme une bonne affaire», malgré un manque total d'information à propos de l'exportation ;

- une phase d'intérêt pour l'exportation qui se traduit par un comportement actif et intentionnel de la part du dirigeant ;

- une phase d'intention d'exporter, phase durant laquelle le dirigeant « adopte un comportement actif à l'égard de l'export », c'est-à-dire qu'il « applique mentalement à la situation présente et future sa décision d'exporter»;

- une phase d'essai durant laquelle «le dirigeant intensifie notamment sa recherche d'information sur les marchés étrangers et évalue les capacités de son entreprise dans l'optique d'une stratégie d'exportation».

À notre avis, la contribution de notre recherche se situe également dans les prolongements que l'on peut en faire, en particulier en termes d'outil d'aide à la décision d'internationalisation. Le but poursuivi par un tel travail était de permettre aux dirigeants de petite entreprise de mener une réflexion sur les cohérences des choix industriels et organisationnels à opérer pour construire la capacité de réponse de leur entreprise à des marchés étrangers et la formulation de ces cohérences (entre choix industriels et organisationnels) leur permettant de concevoir partiellement leur processus d'internationalisation. Ce travail semble largement se justifier par l'intensification des échanges internationaux et la difficulté de la petite entreprise à «tirer son épingle du jeu ». Néanmoins, beaucoup plus importante, cette tâche nécessite, à notre avis, un complément pour mettre en évidence des variables structurant et permettant de juger du processus d'internationalisation de la PME/PMI.

En ce qui concerne la méthode de recherche retenue, nous avons tenu compte des conclusions de nombreux chercheurs selon lesquelles une étude longitudinale permettrait de valider (et d'expliquer) l'enchaînement des phases du processus 


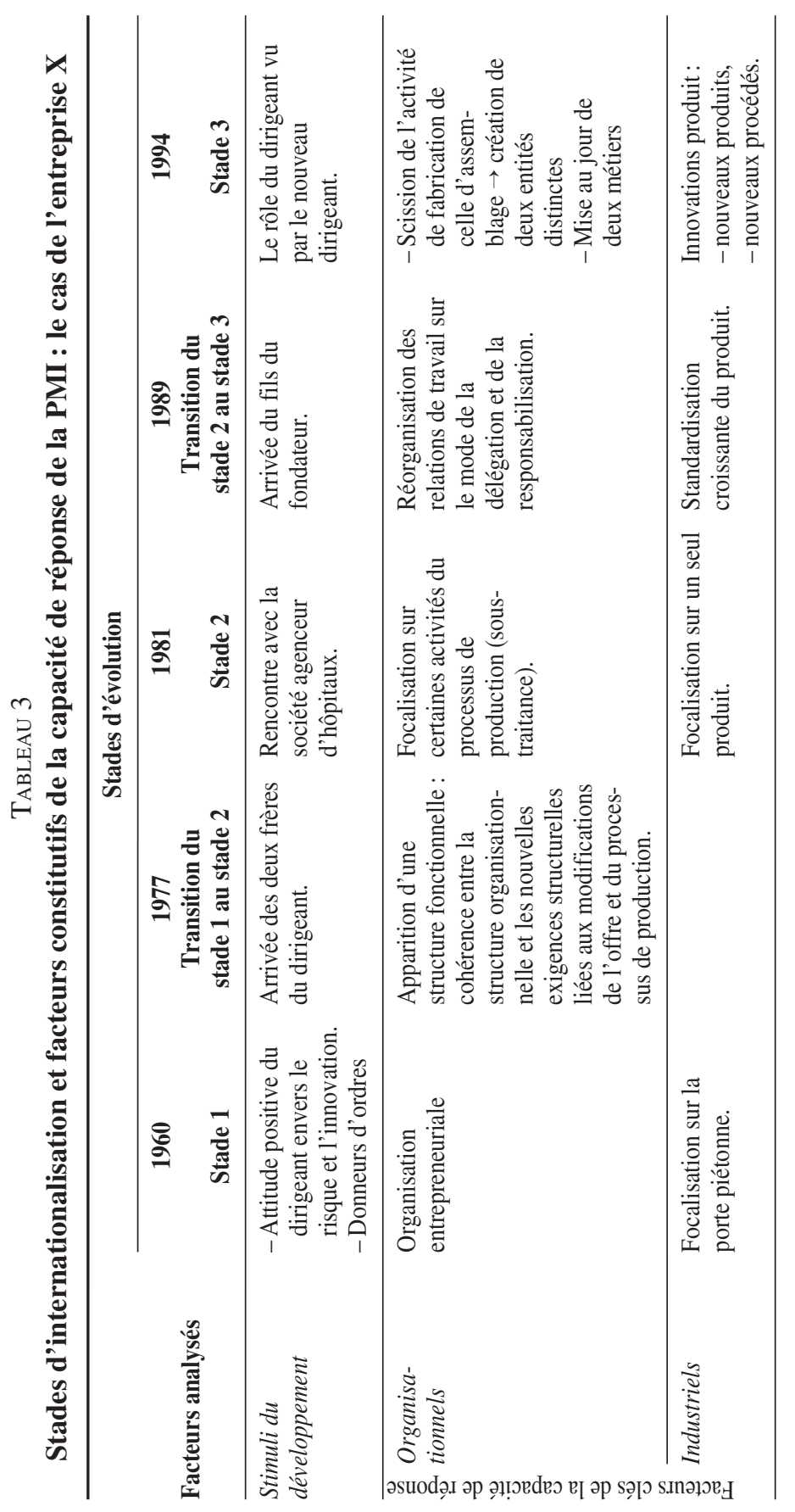

Revue internationale P.M.E., vol. 14, n 2, 2001 


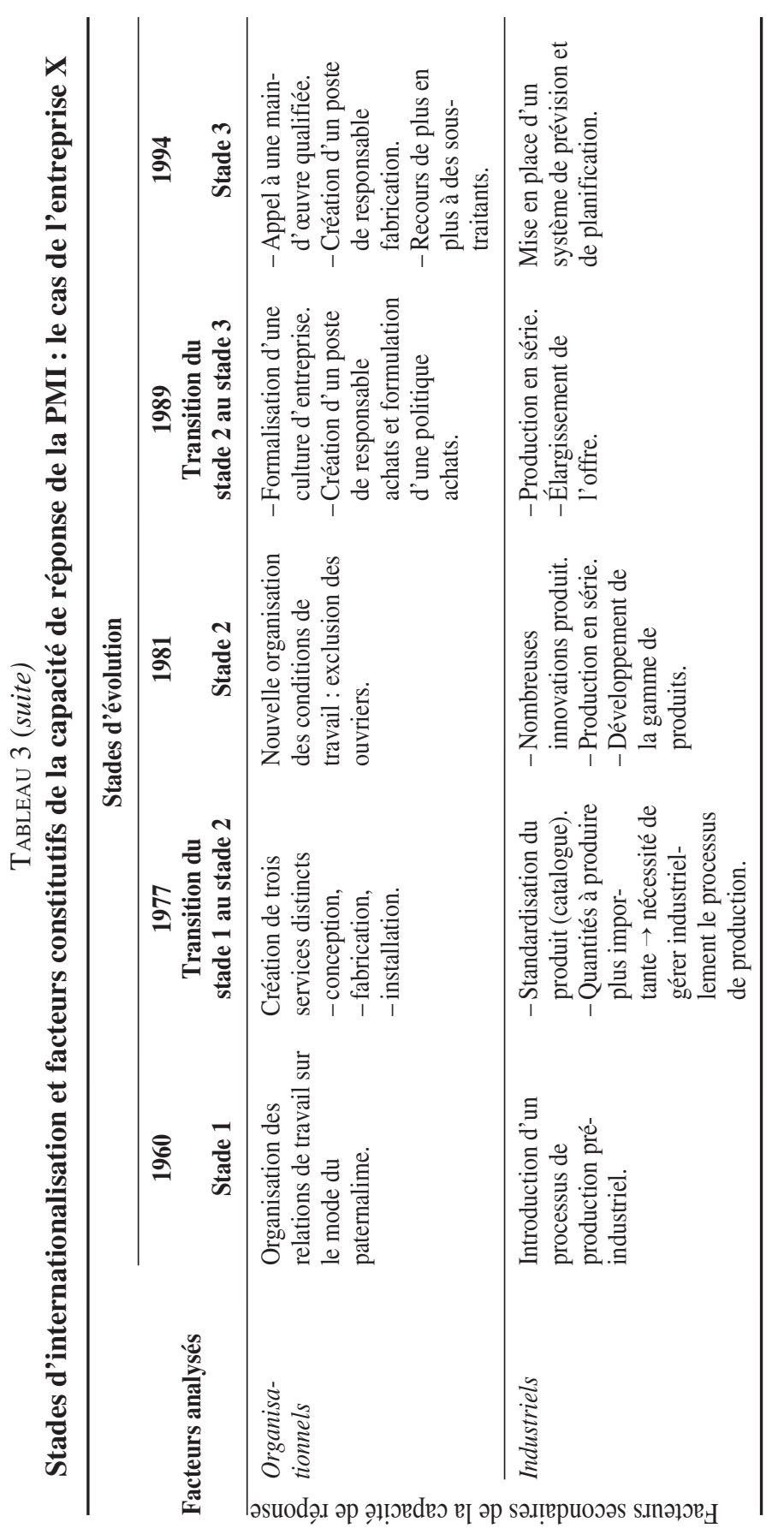

Revue internationale P.M.E., vol. 14, $\mathrm{n}^{\circ}$ 2, 2001 
d'engagement à l'export. Pourtant, une étude longitudinale ne peut se réduire à une simple juxtaposition d'études menées à des dates et à des périodes distinctes, mais requiert la conduite de nombreux entretiens pour reconstituer une histoire de l'entreprise aussi fidèle que possible, même si nous restons consciente des nombreux biais qu'implique une telle analyse. En effet, le recueil d'information par entretiens, parce qu'il s'appuie sur des discours d'acteurs à propos d'événements passés comporte deux biais majeurs. Le premier concerne la mémoire et l'oubli, et le second, les problèmes d'interprétation et de jugement. L'existence de ces biais nous a d'ailleurs amenée à diversifier nos sources d'information en procédant à des entretiens auprès de différentes personnes, et ce, à travers des récits historiques (pour connaître l'histoire telle qu'elle s'est réellement passée dans l'entreprise). Cette double précaution nous a permis d'établir des croisements et des recoupements qui nous ont permis de réduire, sans les éviter, certains de ces biais.

Cet article visait à démontrer que les modèles sur la théorie de l'internationalisation de la PMI, loin d'être dépassés, représentent une recherche substantielle dans le domaine de l'internationalisation malgré leurs nombreuses limites (l'internationalisation ne peut se réduire à la seule exportation et elle n'est pas toujours un processus séquentiel, ni un processus prédéterminé et ordonné, ni un processus irréversible).

\section{Bibliographie}

Ageron, B. (1999), Le processus d'internationalisation de la PMI : Un outil d'aide à la conception (et au pilotage), Thèse de doctorat, IAE de Lyon.

Ageron, B. et C. Pellegrin (1996), «Les conditions préalables au sein des PMI rhônalpines, aux processus de modernisation et internationalisation : Cohérence entre logiques sociales et logiques industrielles », Rapport région Rhône-Alpes.

ALGOE (1993), PMI 93, Rapport détaillé, Ministère de l'Industrie, des Postes et Télécommunications et du Commerce extérieur, Commissariat général du Plan, 527 p.

ANDERSEN, O. (1993), « On the international process of firms : a critical analysis », Journal of International Business Studies, 2e trimestre, p. 209-231.

BILKEY, W.J. et G. TESAR (1977), « The export behavior of small Wisconsin manufacturing firms », Journal of International Business Studies, vol. 9, nº 1, p. 93-98.

BONACCORSI, A. (1992), «On the relationship between firm size and export intensity», Journal of International Business Studies, 4 e trimestre, p. 605-635.

CAvusgil, S.T., W.J. BiLkeY et G. Tesar (1979), «A note on the export behaviour of firms export profils », Journal of International Business Studies, vol. 10, nº 1, p. 91-97.

CAvusgil, S.T. (1980), «On the internationalisation process of firms », European Research, vol. 8, p. 273-281.

Revue internationale P.M.E., vol. 14, $\mathrm{n}^{\circ}$ 2, 2001 
CAVUSGIL, S.T. (1982), «Some observations on the relevance of critical variables for international stages », dans M.R. Czinkota et G. Tesar, Export Management : An International Context, New York, Praeger.

CRICK, D. (1995), « An investigation into the targeting UK export assistance », European Journal of Marketing, vol. 29, no 8, p. 76-94.

CzInKota, M.R. (1982), Export Development Strategies : US Promotion Policies, New York, Praeger.

DALLI, D. (1994), «The exporting process: the evolution of small and medium-sized firms », Advanced in International Marketing, vol. 6, p. 85-110.

DEBOURSE, J.P. et J. VASSEUR (1985), « Réflexions sur l'étude du processus d'internationalisation », Communication aux VII ${ }^{\text {es }}$ Journées nationales des IAE, Lille, 10 p.

Dichtl, E., F. Kondo, H.G. KoleglmayR et S. Muller (1984), «The foreign orientation of managers : a key variables - export promotion and export results », The Ritsumeikan Business Review, Kyoto, vol. 23, no 2, p. 1-33.

FANKAM, J.M. (1998), «Les déterminants de la planification de l'exportation en PMI », Communication à la VIII ${ }^{\mathrm{e}}$ Conférence de l'AIMS, Bruxelles.

FORD, D. et L.C. LEONIDOU (1991), « Research development in international marketing: a European perspective», dans S.J. Paliwoda, New Perspectives in International Marketing, Londres, Routledge, p. 3-32.

GiBIAT, M. (1994), «Les modèles intégrés de la décision d'exporter en PME/ PMI : synthèse des recherches depuis 20 ans », Revue internationale PME, vol. 7, $\mathrm{n}^{\circ}$ 2, p. 11-29.

Huberman, M.A. et M.B. Miles (1991), Analyse de données qualitatives - Recueil de nouvelles méthodes, Bruxelles, De Boeck University.

INTERSTRATOS (1995), EIASM Institute Report, European Institute for Advanced Studies in Management, juillet, $122 \mathrm{p}$.

JOHANSON, J. et J.E. VAHLNE (1977), «The internationalization process of the firm: a model of knowledge development and increasing foreign market commitment», Journal of International Business Studies, vol. 8, n 1, p. 23-32.

JOHANSON, J. et F. WIEDERSHEIM-PAUL (1975) «The internationalization of the firms : four Swedish cases », Journal of Management Studies, octobre, p. 305-322.

Julien, P.-A., P.-Y. LÉO et J. PHILIPPE (1995), PME et grands marchés, Paris, L'Harmattan.

JULLS, M. et P. WATERS (1987), «The internationalization of Norvegian firms : a study of the UK experience », Management International Review, vol. 27, nº 1, p. 58-66.

KALIKA, M. (1986), «La structuration par l'internationalisation », Revue française de gestion, janvier-février, p. 77-84.

Kalika, M. (1988), Structures d'entreprises : déterminants, réalités et performances, Paris, Economica.

KAMATH, S., P.J. Rosson, D. PATTON et M. BROOKS (1987), «Research on success in exporting : past, present and future », dans J. Rosson et S.D. Reid, Managing Export Entry and Expansion, New York, Praeger, p. 398-423.

Revue internationale P.M.E., vol. 14, n 2, 2001

(C) 2001 - Presses de l'Université du Québec

Édifice Le Delta I, 2875, boul. Laurier, bureau 450, Sainte-Foy, Québec G1V 2M2 • Tél. : (418) 657-4399 - www.puq.uquebec.ca

Tiré de : Revue internationale P.M.E., vol. 14, $\mathrm{n}^{\circ} 2$, sous la direction de Pierre-André Julien. 
KAYNAK, E. et V. KOTHARI (1984) «Export and behavior of small sized manufacturers : some policy guidelines for international marketers », Management International Review, vol. 24, no 2, p. 61-69.

KENG, K.A. et T.S. JIUAN (1989), « Differences between small and medium sized exporting and non-exporting firms : nature or nurture», International Marketing Review, vol. $6, n^{\circ} 4$, p. $27-40$.

KLEINSCHMIDT, E. et R.G. CoOPER (1984), «A typology of export strategies applied to the export performance of industrial firms », dans E. Kaynak, International Marketing Management, New York, Elsevier Science Publishing Co., p. 217-231.

LAngston, C.M. et K.R. TEAs (1976), «Export commitment and the characteristics of management», Communication au congrès annuel de la Midwest Business Association.

LANZARA, R. (1987), « Strategic differenciation and adaptation among small- and mediumsized Italian exporting manufacturers », dans J. Rosson and S.D. Reid, Managing Export Entry and Expansion, New York, Praeger, p. 41-53.

LEONIDOU, L.C. et C.S. KATSIKEAS (1996), « The export development process : an integrative review of empirical models », Journal of International Business Studies, $3^{\mathrm{e}}$ trimestre, p. 517-551.

MIESENBOCK, K.J. (1988), «Small businesses and exporting : a literature review », International Small Business Journal, vol. 6, nº 2, p. 42-61.

Millington, A.I. et B.I. BAYliss (1990), «The process of internationalization : UK companies in the EC», Management International Review, vol. 30, n 2, p. 151-161.

MoON, J. et H. LEE (1990), « On the international correlates of export stage development : an empirical investigation into the Korean electronics industry », International Marketing Review, vol. 7, $\mathrm{n}^{\circ}$ 5, p. 16.26.

O'Rourke, A.D. (1989), «Differences in exporting practices, attitudes and problems by size of firm », American Journal of Small Business, vol. 9, nº 3, p. 25-29.

REID, S.D. (1981), «The decision-maker and the export entry and expansion », Journal of International Business Studies, vol. 12, n 2, automne, p. 101-112.

REID, S.D. (1982), « The impact of size on export behaviour in small firms », dans M.R. Czinkota et G. Tesar, Export Management : An International Context, New York, Praeger, p. 18-3.

REID, S.D. (1983), « Managerial and firm influences on export behavior », Journal of the Academy of Marketing Science, vol. 11, no 3, p. 323-332.

REID, S.D. et P.J. Rosson (1987), « Managing export entry and expansion : an overview », dans J. Rosson et S.D. Reid, Export Entry and Expansion, New York, Praeger, p. 3-21.

Robinson, R.B., M.Y. SAlem, J.E. Logan et J.A. PeArCe (1986), «Planning activities related to independant retail firm performance », American Journal of Small Business, vol. 11, no 1, p. 19-27. 
Roux, E. (1987), Les facteurs explicatifs de la décision d'exporter: rôle de l'attitude du dirigeant envers le risque, Thèse de doctorat, Aix-Marseille.

Tarondeau, J.C. (1993), Stratégie industrielle, Paris, Vuibert, coll. « Gestion».

TESAR, G. et J.S. TARLETON (1982), «Comparison of Wisconsin and Virginia small and medium-sized exporters : agressive and passive exporters », dans M.R. Czinkota et G. Tesar, Export Management: An International Context, New York, Praeger.

WELCH, L.S. et R. LUOSTARINEN (1988), «Intemationalization : evolution of a concept », Journal of General Management, vol. 14, $\mathrm{n}^{\circ}$ 2, p. 34-55.

WorTZEL, L. et H. WORTZEL (1981), « Export marketing strategy for NIC and LDC based firms », Columbia Journal of World Business, printemps, p. 51-60.

YAPRAK, A. (1985), «An empirical study of the differences between small exporting and non-exporting US firms », International Marketing Review, vol. 2, nº 2, p. 72-83.

YIN, R.K. (1994), Case Study Research : Design and Methods, Thousand Oaks, Californie, Sage Publications.

YounG, S. (1987), «Business strategy and the internationalization of business : recent approaches », Managerial and Decision Economics, vol. 8, p. 31-40.

Revue internationale P.M.E., vol. 14, n 2, 2001 